\title{
Prosodic processing in sentences with 'only' in L1 and L2 English
}

\author{
Rachida Ganga ${ }^{1}$, Haoyan $\mathrm{Ge}^{2}$, Marijn E. Struiksma ${ }^{1}$, Virginia Yip ${ }^{3}$, and Aoju Chen ${ }^{1}$ \\ ${ }^{1}$ Utrecht Institute of Linguistics OTS, Utrecht University, The Netherlands \\ ${ }^{2}$ School of Education and Languages, The Open University of Hong Kong, China \\ ${ }^{3}$ Department of Linguistics and Modern Languages, The Chinese University of Hong Kong, \\ China
}

Corresponding author: Rachida Ganga (r.ganga@uu.nl)

\begin{abstract}
It has been proposed that processing in the native language and in the second language differ because the second language learners experience influence from their native language or are unable to integrate information from multiple linguistic domains. Different methodologies have provided evidence for either account, such is the case for advanced Dutch learners of English. They have been considered native-like in their offline comprehension but appear to experience difficulties during online processing. To shed more light on the underlying mechanism, we examined their processing of sentences with only, which require the processing of the prosodic-semantic-syntactic interface. Using an event-related potentials (ERP) paradigm to map rapid integration processes, the current study examined the differences in the processing and integration of prosody with semantic and syntactic information in English sentences with only between native speakers of English and advanced Dutch learners of English. We found that Dutch listeners processed accentuation adjacent to only in a native-like manner but not accentuation further in the sentence. The Dutch listeners had a default interpretation of sentences with only, but were unable to update this when it was not true. Thus, they failed to integrate information to adjust their expectation for information later in the sentence.
\end{abstract}

Keywords: prosodic processing, focus particle only, contrastive accentuation, expectancy, accent positivity, P600, second language acquisition

\section{Introduction}

There has been much discussion on why processing in the native language (L1) and in the second language (L2) differ from each other, but there has not yet been a consensus. On the one hand, it has been suggested that knowledge from the L1 may influence how the L2 is processed (Clahsen \& Felser, 2006; Frenck-Mestre, 2002; Juffs, 2005). Prior knowledge from the L1 facilitates or hinders the comprehension of linguistic constructions in a different language. If these constructions are similar in both languages, L2 learners use a similar processing strategy that they use in the L1 to process the L2, making them quicker or more accurate when coming to the correct interpretation. If the constructions are not similar, however, the L2 learner has to employ different processing strategies, resulting in slower or inaccurate processing. On the other hand, L2 learners may experience difficulties during the integration of information when it involves the interface between certain linguistic domains (Sorace, 2011; Sorace \& Filiaci, 2006). The latter view, the failure to integrate, suggests that L2 learners are able to acquire the simple syntactic structures, but they cannot combine information from multiple constructs and adjust their interpretation as the sentence unfolds (Sorace, 2011; Sorace \& Filiaci, 2006). Although not all interfaces may form a problem for L2 learners (White, 2011), it is suggested that L2 learners may have less detailed or less automatically available knowledge, or simply fewer cognitive resources available for the integration of problematic interfaces (for a review see Sorace, 2011). The studies by Juffs (2005) and Marinis, Roberts, Felser, and Clahsen (2005) are examples of studies that provide evidence for either point of view. Juffs (2005) subjected Spanish, Japanese, and Chinese L2 learners of English to a self-paced reading task. They judged the grammaticality or ungrammaticality of sentences that contained $w h$-fronting. This is a syntactic construction that occurs in Spanish, but not in Japanese 
or in Chinese. Although all L2 learners showed a similar pattern of mistakes in their judgements, the Spanish learners were overall more accurate than the other two groups. The knowledge that the Spanish learners had from $w h$-fronting in their L1 may have benefited their judgments of such sentences in their L2, which the Japanese and Chinese learners lacked. Marinis, et al. (2005) tested native speakers of English and L2 learners of English from various L1 backgrounds (Greek, German, Chinese, and Japanese) in a self-paced reading task. Sentences were broken up in chunks and presented in a moving window. As a proxy for online processing, reading time per moving window was tested. The stimuli were English sentences that contained long-distance wh-dependencies. To help parsing, one of the conditions introduced a gap in between clauses. Wh-dependencies can also be found in Greek and German, but not in Chinese and Japanese. If L1 transfer had occurred, Greek and German learners would be facilitated by their L1 and use the gap, whereas Chinese and Japanese learners would not. However, different from the study by Juffs (2005), all L2 learners in this study behaved similarly to each other in that they did not use the gap during parsing, whereas the English listeners did. Therefore, it was not the L1 that influenced L2 processing but, as the Marinis et al. proposed, it was the limited availability of syntactic information during real time processing.

In the same study, Marinis et al. (2005) also examined the learners' offline comprehension. Despite the use of an alternative processing strategy, the L2 learners' comprehension was as accurate as the native speakers'. This demonstrates how offline and online measures can provide diverging results on L2 processing and, by extension, provide contrasting evidence that fits either the L1 influence view or the failure to integrate view (for a review see Clahsen \& Felser, 2006). This is even the case for L2 learners that achieved high proficiency in a L2 that is linguistically similar to their L1. For example, highly advanced Dutch learners of English have shown to be native-like in their comprehension but to be unable to use or integrate information efficiently enough to predict L2 English prosodic contrast online (Akker \& Cutler, 2003; Chen \& Lai, 2012; Ge, Chen, \& Yip, 2018). Examining these advanced Dutch learners of English provides the opportunity to further investigate the mechanism behind poor online sentence processing despite high proficiency in the L2. Specifically by examining their processing of sentences with only. Such sentences exist in both Dutch and English and require complex processing of the interface between the prosodic, semantic, and syntactic domain (more on this interface in Section 1.1, and the processing of the interface in Section 1.2). Previous offline and online processing studies have shown that advanced Dutch learners of English understand L2 sentences with only in a native-like manner offline (Ge et al., 2018), but they do not process prosodic information to rapidly update sentence interpretations online (Ge et al., in prep.).

To understand why prosodic information is not processed in a native-like manner, more insight is needed into how Dutch learners of English process and integrate prosodic information with information from the rest of the sentence. The current study examined the L2 processing of English contrastive pitch accents and their integration with the semantic and syntactic information in sentences with only using an online measure to map the cognitive responses as the pitch accent unfolds, namely electroencephalogram (EEG).

EEG forms the ideal method to answer how one processes pitch accents and how they use information from the semantic and syntactic domain to adjust this processing in real-time. For example, it has been shown with EEG that L2 learners are sensitive to contrastive pitch accents but use them differently than native speakers, e.g. for lexical processing instead of processing of the information structure (Lee, Perdomo, \& Kaan, 2019). EEG uses electrodes to pick up and record electrical information that is generated by mass activation of neurons in the brain, a reflection of cognitive processing. It accurately displays brain activation over time with only a delay of a few milliseconds and thus representing real-time processing. This is different from eye-tracking, which has a relatively long delay between the processing in the brain and the behavioral output, the eye-movement, due to the brain's need to plan and execute the motor movement in addition to processing language. This high temporal resolution of EEG opens up the possibility to describe how and when the brain responds to a stimulus. This is especially the case when using the event-related potentials (ERP) paradigm. In this paradigm, the onset of the stimulus, in our case the onset of the word that is accented is aligned with the onset of the brain response. It allows for the millisecond by millisecond representation of how the brain builds expectations and the sentence meaning as it is incorporating information from only and the pitch accent.

The rest of the paper is structured as follows. To understand the difficulties that L2 learners face when processing sentences with only in the L2, we first go over its semantic, syntactic, and prosodic properties in Dutch and English in Section 1.1. In Section 1.2., we provide evidence from studies on online processing of prosodic information in the L1 and L2. In Section 1.3, we discuss ERP research on sentences with only to build general and ERP specific hypotheses. We then present our research questions and hypotheses. After which we discuss the methods, analyses, and results. Finally, we conclude with how our findings add to the current knowledge on L2 processing.

\subsection{The prosodic-semantic-syntactic interface in sentences with only}

Sentences with only contain prosodic, semantic, and syntactic properties that affect the listener's processing. Semantically, only signals an upcoming contrast in the utterance. It flags characteristics of the contrasted element as true, and activates alternatives that are related to the contrast but have characteristics that are not true (Filik, Paterson, \& Liversedge, 2009; Zimmermann \& Onea, 2011). This contrast is signaled by prosodic means, i.e. accentuation in both English and Dutch (Gussenhoven, 1983; Ladd, 1980). This accentuation highlights the locus of the contrastive focus (Zimmermann \& Onea, 2011). In example (1a), the element that is accented is ball, eliciting an object focus reading. Only highlights that the ball is the one thing that John is throwing, and that he is not throwing any other (alternative) throwable object. In (1b), the accented element, throwing, triggers a verb focus reading. Throwing is the one action that John does to the ball and nothing else. 
(1) a. John is only throwing the BALL, not throwing the pen. b. John is only THROWING the ball, not kicking the ball.

(object focus)

(verb focus)

English and Dutch speakers both use only (or the Dutch equivalent alleen) in combination with pitch accents to construct sentences with only. However, they differ in how much they rely on the prosodic features to encode the locus of contrastive focus as result of a differences in the syntactic construction of sentences with only. In English, verbs are preferably placed adjacent to their direct objects (Bouma, Hendriks, \& Hoeksema, 2007). As a result, only is often put in a preverbal position (e.g. as depicted in 1a and 1b). Pitch accents then become the primary cue to locate the focus. In Dutch however, alleen 'only' is preferably positioned adjacent to its focal element ( $2 \mathrm{a}$ and $\mathrm{b}$ ) (Bouma et al., 2007; Foolen, Gerrevink, Hogeweg, \& Prawiro-Atmodjo, 2009). Therefore, in addition to the accentuation, the positioning of only also encodes the locus of focus in Dutch.

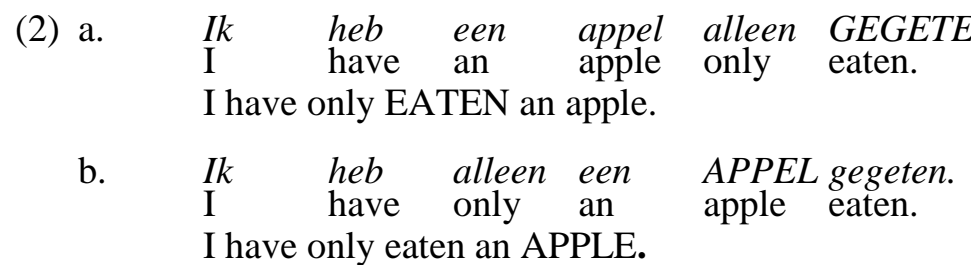

\subsection{The online prosodic processing of sentences with only in the L1 and L2}

Both Dutch and English listeners use pitch accents to interpret focus in sentences with only differently in their L1 (Ge et al., in prep.; Mulders \& Szendroi, 2016). Mulders \& Szendroi (2016) tested the focus processing of L1 Dutch in sentences as in (3), using the visual world paradigm in an eye-tracking experiment. The visual stimuli included pictures that were in accordance or in disagreement with the auditory stimuli. For example, a picture matching (3a) contained a fireman who was holding a plate with celery and an empty plate. A mismatching picture depicted a fireman who was holding a plate with celery and a plate with another item.

(3) Example stimuli from Mulders \& Szendroi (2016, p5).

$\begin{array}{lllll}\text { a. Ik heb alleen SELDERIJ aan de } & \text { brandweerman } & \begin{array}{l}\text { gegeven. } \\ \text { given. }\end{array} \\ \text { I theman } & \text { have only celery to the } \\ \text { b. I only gave CELERY to the fireman. } & & & \\ \text { b heb alleen selderij aan de } & \text { BRANDWEERMAN } & \begin{array}{l}\text { gegeven. } \\ \text { given. }\end{array}\end{array}$

Mulders \& Szendroi found that the participants used prosodic cues to not only interpret sentences with only but also to build expectations of the sentence meaning as the sentence unfolds. The prosodic focus on the direct object guided where they looked at the moment they heard the indirect object, which corresponded to their interpretation of the utterance. The authors suggested that the listeners quickly interpreted the focus and that the looking behavior at the indirect object was a verification of the sentence meaning. Furthermore, the focus induced anticipatory looks measurable before the indirect object. For example in (3a), the participants looked more at the picture that corresponded to their interpretation: the fireman who was holding celery and nothing else. If there were only mismatching pictures that did not correspond to the sentence, the participants first looked at someone else with only celery before hearing the indirect object. When they heard the indirect object, the participants looked more towards the incorrect image of the fireman holding two items.

The focus processing of sentences with only in L1 English was examined by Ge et al. (in prep.) using a visual world paradigm in an eye-tracking experiment. The auditory stimuli included a story (5a) that introduced a set of verbs and objects, and a target sentence that contained only with either a prosodic verb focus (5b), or prosodic object focus (5c). The visual stimuli included images that contained the subject performing various acts: e.g. a target action, and the alternatives of focus. As an example in (5b and c), the target action was "carrying the bucket". The alternative of the verb focus was "throwing the bucket" (5b) and the alternative of the object focus was "carrying the suitcase" (5c). Ge et al. (in prep.) found that L1 English listeners used prosodic information to adjust their interpretation. They looked at the image that depicted the alternative to the focal word at "not".

(5) Example stimuli from (Ge et al., in prep.). a. The dinosaur has a bucket and a suitcase. He was going to carry them and
throw them. Then changed his mind.
b. $\quad$ The dinosaur is only CARRYING the bucket, not throwing the bucket.
c. The dinosaur is only carrying the BUCKET, not carrying the suitcase.

In the same eye-tracking study, Ge et al. (in prep.) also examined the L2 focus processing of sentences with only. Previously, the authors found that highly proficient Dutch learners of English were native-like in their offline comprehension of sentences with only similar to (5b and c) (Ge et al., 2018). In the eye-tracking experiment, Ge et al. (in prep.) aimed to find whether there are differences in if and how quickly L1 English listeners and the Dutch learners of English use prosodic information to come 
to the correct interpretation. The authors found that the Dutch learners of English may not have used prosodic information to alter their interpretation, or they have done so more slowly than the L1 English listeners. Instead of looking more at the alternative of the focus at the "not", Dutch listeners looked more at the image that displayed the target action. Only later in the sentence, at the moment they heard the verb in the "not" sentence, did they shift more towards the alternative of focus.

Although Dutch and English listeners use prosodic cues to adjust their interpretation of sentences with only in their L1 and Dutch learners of English seem to be able to do so native-like during offline comprehension, the question remains why Dutch learners of English display difficulties during online processing in sentences with only. From the L1 transfer point of view, the Dutch learners of English may have projected the focus of only to the element that is directly adjacent to it, similar to their preference to place only adjacent to the focal element (Bouma et al., 2007; Foolen et al., 2009). From the failure to integrate point of view, the Dutch learners of English may have first projected the focus adjacent to only as a default interpretation. When they were confronted with the prosodic information later in the sentence, instructing them that a change in focus interpretation and a reinterpretation of the sentence meaning is needed, they may have been unable to do so in real-time, although this did not affect their comprehension. In the latter case, it is not L1 transfer but the inability to adjust and process in rapid manner. To differentiate between these two hypotheses, more detailed information is needed on how the pitch accents are processed and integrated with the semantic and syntactic information from only. Evidence for such processing and integration can be provided with EEG.

The processing of contrastive pitch accents in L1 Dutch and how only affects this processing has been examined with EEG (Dimitrova, 2012). Dutch participants were presented with auditory stimuli (e.g. Hij heeft alleen water na de training gedronken. 'He drank only water after the training') in four conditions. These conditions differed in whether there was alleen 'only' (5a and b) or not (5c and d), and whether the pitch accent was on the direct object ( $5 \mathrm{a}$ and $\mathrm{c})$, which was adjacent to alleen 'only', or the prepositional object ( $5 \mathrm{~b}$ and $\mathrm{d}$ ), which was further away. The EEG signal was analyzed at the direct (water) and at the prepositional object (training).

(5) Example stimuli (adapted from Dimitrova, 2012a)
a. He drank only WATER after the training.
b. He drank only water after the TRAINING.
c. He drank WATER after the training.
d. He drank water after the TRAINING.

The results from this study provides evidence that there is a similarity in how sentences with only are constructed in Dutch and how only induces expectations that affect the processing of the pitch accent. First, only modified the processing of the contrastive pitch accent; Different processing patterns were found between the sentences with and without only. Second, this modification was dependent on the position of the pitch accent in the sentence (Dimitrova, 2012), suggesting an expectancy-based modulation. The processing of the acoustic features of the pitch accent on the direct object, adjacent to only, was altered, but not when it was on the prepositional object. Furthermore, the processing of the acoustic features on both positions were preceded by an early expectancy processing, which suggests that both pitch accents were expected. However, the polarity of the expectancy patterns differed which may be a result of how the expectations were built (Dimitrova, 2012). The pitch accent on the direct object could only be expected as a result of only, whereas the expectation of the pitch accent on the prepositional object was built with additional information about the accentuation status of the direct object; If the direct object was not accented, the listeners knew that the prepositional object would be. Finally, only after the accentuation of the prepositional object, further away from only, the sentence had to be re-evaluated. This suggests that the listeners had already constructed a possible meaning of the sentence. The pitch accent at the prepositional object provided enough information to conclude that their initial interpretation was incorrect and that re-analysis of the sentence was needed.

\subsection{The current study}

Given the difference in how Dutch and English speakers construct sentences with only and the similarities between the production and processing of its prosodic focus in L1 Dutch, we further examined the difficulties that Dutch learners of English face during online processing of English sentences with only. The current ERP study investigated the differences in the processing of prosodic focus between L1 English listeners and highly proficient Dutch learners of English. Our goal was to provide additional evidence for either the $\mathrm{L} 1$ point of view or the failure to integrate prosodic information with only's semantic and syntactic information.

Although native-like in their comprehension, highly advanced Dutch learners of English do not use or integrate information efficiently enough to predict L2 English prosodic contrast (Akker \& Cutler, 2003; Chen \& Lai, 2012; Ge et al., 2018, in prep.). With this in mind, we hypothesized that Dutch learners of English in our study would have difficulty in the integration of information. We expected that English and Dutch listeners would differ in their processing of prosodic patterns. As there has not yet been an EEG study on L1 English focus processing using sentences with only, we used Dimitrova (2012)'s EEG study on L1 Dutch prosodic processing in sentences with only as a foundation for ERPspecific hypotheses. When hearing the prosodic focus adjacent to only, we expected that English listeners show an early positive expectancy peak, followed by the processing of the acoustic features of the pitch accent. In the case of nonadjacency of focus, we expected English listeners to show an early negative expectancy peak, followed by the processing of the pitch accent's acoustic features, and finally the re-evaluation of the sentence meaning. For the Dutch listeners, we expected that they would fail to show such expectancy and sentence re-evaluation processing patterns. As there has been evidence that the availability of a discourse context facilitates prosodic processing in L1 Dutch listeners (Dimitrova, 
Stowe, Redeker, \& Hoeks, 2012), we presented the sentences with only in isolation and in a context setting that presented alternatives to verbs and objects but did not specifically point towards the contrast. We expected that Dutch learners would be facilitated by the context in that they would be able to process the acoustic features of the pitch accents more easily than in isolated sentences.

\section{Materials and methods}

\subsection{Participants}

Thirty-seven native speakers of Dutch (14 male, 19-26 years old, $\mathrm{M}=22$ years) (from now on the L2 English group) and twenty native speakers of English (8 male, 18-34 years old, $M=24$ years) (from now on the L1 English group) participated after signing a written informed consent in accordance with the Ethics assessment Committee Linguistics (ETCL) of the Utrecht Institute of Linguistics.

Because of the difficulty in finding native speakers of English in the Netherlands, we included native speakers of various English varieties to increase the total number of native participants. We acknowledge that English varieties differ in their use of prosody which may skew our results. However, we lacked the statistical power to test whether English variety did affect prosodic processing. The L1 English group consisted of participants from the United States $(n=8)$, England $(n=7)$, Ireland $(n=1)$, Australia $(n=2)$, Canada $(n=1)$, and a British community in Hong Kong who did not have knowledge of Mandarin Chinese or Cantonese $(n=1)$.

Two female L1 English participants were excluded due to failure to reach $80 \%$ correct responses on the semantic relatedness task to assess attention. Four Dutch speaking participants (2 male) were excluded due to technical failure $(n=2)$, being left-handed $(n=1)$, or failure to reach $80 \%$ correct responses on the semantic relatedness task $(n=1)$. The remaining participants were right handed, had normal or corrected to normal vision, and reported not having any neurological, psychiatric, hearing, or language impairments. Furthermore, the L2 English group was considered highly proficient in English by their scores $(M=87.88 ; \mathrm{SD}=9.97$ ) on the LexTALE (Lemhöfer \& Broersma, 2012). Because of the relatively high and consistent scores, we did not include proficiency in the statistical analyses.

\subsection{Materials}

We used auditory stimuli from Ge et al. (2018, in prep.)'s studies on L1 and L2 processing of English sentences with only. We modified the stimuli so that the listeners relied more on prosody to resolve ambiguity. For example the target sentence 'The dinosaur is only CARRYING the bucket, not throwing the bucket' was edited to 'The dinosaur is only CARRYING the bucket'. The latter sentence allows for the correct interpretation only if the pitch accent is interpreted and integrated correctly. By using similar stimuli to Ge et al. (in prep.)'s study, we were able to compare our results with their findings.

Ge et al. (2018, in prep.) recorded a native male speaker who produced sixty unique pairs of naturalistic British-English stimuli $(\mathrm{n}=120)$ at $44.1 \mathrm{kHz}$ sampling frequency with 16 bits resolution in a recording booth. The speaker was instructed to produce half of these 120 stimuli with context and verb accentuation ( $\mathrm{A}$ in Table 1 ), and the other half with context and object accentuation as natural as possible (B in Table 1). The context sentences were not meant to provide a clear contrastive element. Instead, their function was to present possible alternatives to the verbs and the objects and to raise the expectation for any contrast. We removed the context sentences to produce 120 additional stimuli (C and $D$ in Table 1) that were otherwise acoustically identical to the set with context (i.e. $C$ was acoustically identical to A and D to B in Table 1). In total, we used 240 experimental items.

Table 1. Examples of experimental materials in British-English

Context sentences

The dinosaur has a

pumpkin and a bucket. He

was going to throw them

and kick them. Then he

changed his mind.
Target sentences

Verb accentuation

(A) The dinosaur is only THROWING the bucket.
Object accentuation

(B) The dinosaur is only throwing the BUCKET.

\section{$-*$ \\ (C) The dinosaur is only \\ (D) The dinosaur is only THROWING the bucket. throwing the BUCKET.}

* Target sentences $\mathrm{C}$ and D are not preceded by context sentences.

In addition to the experimental items, we used Ge et al. (2018, in prep.)'s fillers $(\mathrm{n}=118)$, which were recorded in the same session as the experimental items. We compiled a list of four types of fillers to come up to an appropriate amount for an EEG study. As a result, even though these types all lacked only, there were a few other intrinsic differences. That is, 26 fillers contained context sentences that included an additional object and a target sentence that ended with the confirmation of the action (filler type A in Table 2), 52 fillers were similar to filler type A but did not have the context sentences (filler type B in Table 2), 20 fillers had context sentences with an additional sentence and a target sentence with pitch accent that was congruent to the context (filler type $\mathrm{C}$ in Table 2), 20 fillers were similar to filler type $\mathrm{C}$ but with prosody incongruent to the context sentences (filler type D in Table 2). Despite the variability in the fillers, we believed they were sufficiently different from the experimental stimuli 
to distract the participant from our research purpose. This was confirmed by the answers the participants provided in an informal questionnaire after the experiment.

Table 2. Examples of filler materials.

\begin{tabular}{cll} 
Filler type & Target sentences \\
\hline \multicolumn{1}{c}{ Confirmation of action } & \\
\hline $\mathrm{A}(\mathrm{n}=26)$ & $\begin{array}{l}\text { The dog has a broom, a paper, } \\
\text { and a tomato. He was going to }\end{array}$ & The dog is GETTING the tomato, not \\
& wash the tomato. Then he &
\end{tabular}

$\mathrm{B}(\mathrm{n}=52) \quad$ changed his mind.* $^{*}$

The dog is GETTING the tomato, not squeezing the tomato.*

\begin{tabular}{ll}
\hline Prosodic (in) congruity \\
\hline $\mathrm{C}(\mathrm{n}=20)$ & The fox has a banana and pear. \\
& She was going to peel the \\
banana. Then she changed her \\
mind. I wonder what the fox is \\
peeling.*
\end{tabular}

Twelve lists, each containing all the experimental and filler items, were created so that that all participants listened to all stimuli. Six of these lists were uniquely pseudo-randomized, after which they were reversed for the remaining six lists, i.e. the first trial of the original list became the last trial of the new list. Doing so ensured that the placement of trials within the experiment was counterbalanced across participants and therefore minimized the effect of fatigue in the EEG signal of particular trials. Pseudorandomization ensured that (1) subjects of the sentence were not repeated in adjacent trials, (2) trials within the same condition were not presented more than three times in succession, (3) trials with the same positioning of pitch accents were not presented more than three times in succession, and (4) trials with/without context sentences were not presented more than three times in succession. Each list was preceded by the same practice round consisting of 4 items. Two of the practice items were similar to the experimental items and two were similar to the filler items.

\subsubsection{Acoustic analysis}

To test whether our stimuli were acoustically different, we did an analysis on the acoustic measurements that were retrieved with Praat (Boersma \& van Heuven, 2001). Because we were interested in the processing of the pitch accents, we only tested the critical words (i.e. verbs and objects) on 5 parameters: mean pitch (fO), minimum pitch (fO min), maximum pitch (f0 max), pitch range (fO range), and duration). We expected that accentuation would result in longer duration, higher f0 max, lower f0 min, and a larger f0 range. Our acoustic data were skewed and contained missing data due to differences in the voice quality, so we opted for a Wilcoxon signed-rank test. The results are depicted in Table 3.

We found that accented verbs in comparison to unaccented verbs had a longer duration (410 ms versus $374 \mathrm{~ms}$ respectively), higher fo $(158 \mathrm{~Hz}$ versus $84 \mathrm{~Hz})$, lower f0 min $(72 \mathrm{~Hz}$ versus $73 \mathrm{~Hz})$, higher f0 max $(209 \mathrm{~Hz}$ versus $96 \mathrm{~Hz})$, and a larger f0 range $(134 \mathrm{~Hz}$ versus $22 \mathrm{~Hz})$. Similarly, accented objects in comparison to unaccented objects had a longer duration ( $388 \mathrm{~ms}$ versus $345 \mathrm{~ms}$ respectively), higher f0 $(189 \mathrm{~Hz}$ versus $70 \mathrm{~Hz})$, higher f0 min $(132 \mathrm{~Hz}$ versus $63 \mathrm{~Hz})$, higher f0 max $(217 \mathrm{~Hz}$ versus $77 \mathrm{~Hz})$, and a larger f0 range $(85 \mathrm{~Hz}$ versus $14 \mathrm{~Hz})$. Thus, accentuation resulted in the expected acoustic changes in duration and pitch and could be used for the ERP experiment without further manipulation.

Table 3. Acoustic measurements of verbs and objects.

\begin{tabular}{lllllll} 
& $\begin{array}{c}\text { Accented } \\
\text { verbs }\end{array}$ & $\begin{array}{l}\text { Unaccented } \\
\text { verbs }\end{array}$ & $p^{*}$ & $\begin{array}{l}\text { Unaccented } \\
\text { objects }\end{array}$ & $\begin{array}{l}\text { Accented } \\
\text { objects }\end{array}$ & $p^{*}$ \\
\hline Duration $(\mathrm{ms})$ & $410(48)$ & $374(45)$ & .000 & $345(82)$ & $388(74)$ & .000 \\
F0 (Hz) & $158(19)$ & $84(11)$ & .000 & $70(38)$ & $189(26)$ & .000 \\
F0 min $(\mathrm{Hz})$ & $72(34)$ & $73(15)$ & .008 & $63(39)$ & $132(63)$ & .000 \\
F0 max $(\mathrm{Hz})$ & $209(17)$ & $96(8)$ & .000 & $77(39)$ & $217(24)$ & .000 \\
F0 range $(\mathrm{Hz})$ & $134(41)$ & $22(16)$ & .000 & $14(18)$ & $85(65)$ & .000 \\
\hline
\end{tabular}

Means and standard deviation (in brackets) are presented.

$*$ Significance at $p<0.05$.

\subsection{EEG procedure}

Participants were seated in front of a computer screen. After electrode application and instructions, four practice trials were completed preceding the experiment, which were presented with Neurobehavioral Systems' Presentation (v.20.0 05.20.17). Auditory stimuli were presented via loudspeakers, positioned 
on either side of the computer screen, and were divided over 6 blocks of 51 trials and 1 block of 56 trials. Each block was approximately 11 minutes long and was followed by a 2 -minute forced break. In addition to the forced break, optional breaks were provided approximately every 4 minutes into each block. To minimize eye movement, participants fixated on a white cross against a black background, which appeared $100 \mathrm{~ms}$ before stimulus presentation and remained until $1000 \mathrm{~ms}$ after the stimulus had ended. After each trial, three dashes appeared for $2000 \mathrm{~ms}$ to indicate that blinking was allowed.

In $25 \%$ of the trials $(n=94$, randomly selected $)$, the participants were visually presented with a semantic relatedness task similar to the task presented in Dimitrova et al. (2012)'s L1 Dutch prosodic processing study. This task gauges the participant's attention while minimizing task-related ERPs. Instead of the three dashes, a word was presented visually at the end of a trial. The participants were instructed to decide (forced yes or no) whether the presented word was related to the fragment they last heard. The relatedness of the words and word type (subject, verb, or object) were counterbalanced. After answering the question, the three dashes for blinking appeared for $2000 \mathrm{~ms}$ before continuing to the next trial.

EEG was recorded at $2048 \mathrm{~Hz}$ using a 64-channel BioSemi cap with $\mathrm{Ag} / \mathrm{AgCl}$ electrodes in the 1020 configuration. Additional electrodes on both mastoids, next to both eyes, and above and below the left eye were used for offline referencing, correction of horizontal eye movements (hEOG), and correction of vertical eye movements (vEOG) or blinks respectively.

\subsection{EEG pre-processing steps}

EEG markers were time-locked to the acoustic onset of the critical words, i.e. the verbs and objects. The raw EEG signal was pre-processed and analysed offline using Brain Products' BrainVision Analyzer (v2.1.2.327) and Matlab (R2019a, v.9.6.0.1072779). In BrainVision Analyzer, the data was re-referenced to the average of both mastoids, after which it was bandpass-filtered at 0.1-35 Hz (24 dB slope), downsampled to $500 \mathrm{~Hz}$, and segmented from $-100 \mathrm{~ms}$ to $1000 \mathrm{~ms}$ around the onset of verbs and objects. After segmentation, baseline correction was done by subtracting the mean signal amplitude in the $100 \mathrm{~ms}$ before the word onset from all amplitude values within that segment. Two new channels, $\mathrm{hEOG}$ and vEOG, were created using the hEOG and vEOG electrodes respectively to use in the first round of artefact rejection based on eye movements. Segments that exceeded an amplitude of $\pm 75 \mu \mathrm{V}$ in the hEOG or vEOG channels, displayed a voltage step of $50 \mu \mathrm{V}$ or more between two adjacent sampling points, or in which the difference in signal activity was lower than $0.5 \mu \mathrm{V}$ in an interval of $100 \mathrm{~ms}$, were marked for exclusion from the analysis as a whole segment. In the second artefact rejection step, all 64 scalp electrodes were tested and the channels that exceeded the same rejection criteria were marked for exclusion on an individual basis. Of all trials, 89\% was included in the L1 English group and $87 \%$ in the L2 English group.

Because of the discrepancy between the number of participants in the L1 and L2 English group, we analysed the data on an individual trial basis. This allowed us to increase the number of data points, which in turn increased the power in the statistical analysis. Furthermore, trial-based analysis atlowed us to include all participants whose data would have otherwise been excluded due to the criteria of having too many segments lost to the artefact rejection (e.g. if $>50 \%$ of the trials were rejected). To further examine individual trial data, the data were exported from BrainVision Analyzer to Matlab using the Matlab export solution.

In Matlab, the marked rejected trials were excluded by replacing the amplitude values in each timepoint with NA values on segment level or individual electrode level. The EEG amplitudes within each trial were averaged over three time-windows for each electrode for each word in each condition. We examined the expectancy, the processing of the acoustic features of the pitch accent, and the sentence re-interpretation using pre-defined time-windows based on previous studies on processing contrastive pitch accents instead of visual inspection to minimize multiple comparisons (Luck \& Gaspelin, 2017). These time-windows included an early time-window $(100-200 \mathrm{~ms})$ to test for frontal expectancy patterns, a middle time-window (200-390ms) for global 'accent positivity', and a late timewindow (500-900ms) for the posterior P600. (Dimitrova, Stowe, \& Hoeks, 2015; Dimitrova et al., 2010a; Dimitrova, Stowe, Redeker, \& Hoeks, 2010b; Dimitrova et al., 2012, Regel, Meyer, \& Gunter, 2014). We selected the offset of the middle time-window based on the average duration of the verbs in our stimuli to avoid confounding effects from subsequent words. We also did a visual check to make sure that the objects were not processed differently across conditions as a result of the accentuation status of the preceding verb. To do so, we computed two figures of the object: one with a baseline before the verb and one with a baseline before the object. As both figures looked similar at the acoustic start of the object, we conclude that processing of the objects was not influenced by the accentuation status of the verb across the two conditions ${ }^{1}$.

Finally, the amplitudes of 45 scalp electrodes in each trial were averaged over nine scalp regions based on (Dimitrova, 2012): Left Anterior (Fp1, AF3, AF7, F3, F5, and F7), Left Central (FC3, FC5, C3, C5, CP3, and CP5), Left Posterior (P3, P5, P7, PO3, PO7, and O1), Right Anterior (Fp2, AF4, AF8, F4, F6, and F8), Right Central (FC4, FC6, C4, C6, CP4, and CP6), Right Posterior (P4, P6, P8, PO4, $\mathrm{PO} 8$, and $\mathrm{O} 2)$, Center Anterior ( $\mathrm{FPz}, \mathrm{AFz}$, and $\mathrm{Fz})$, Center Central $(\mathrm{FCz}, \mathrm{Cz}$, and $\mathrm{CPz})$, and Center Posterior (Pz, POz, and Oz). In the end, we had separate datasets for verbs and for objects, each in three separate time-windows. These datasets contained trial data of the EEG signal at respective words in four conditions, that was aggregated over nine scalp regions. 


\subsection{EEG analysis}

Data from each word in each time-window was analyzed separately using mixed-effect modelling in $\mathrm{R}$ with the lmer4 package v.1.1.21 (Bates, Maechler, Bolker, \& Walker, 2015). Interactions were further analyzed with pairwise comparisons using the emmeans package v.1.3.5 (Russell Lenth, 2019). Timewindows for a word were solely analyzed if they stayed within the acoustic boundaries of the respective word. Therefore, the early and middle time-windows were analysed for verbs and objects; and the late time-window only for objects. Models were constructed using the dependent variable ERP and random factors Participant and Item. Fixed factors included Context (with or without), Accent (verb or object), $R O I$ (left anterior, center anterior, right anterior, left central, center central, right central, left posterior, center posterior, right posterior), and Group (L1 English or L2 English). We also added a random slope of Accent in Participants to allow a degree of variability in how each participant responded to the pitch accent. We added factors iteratively and used the -2LL chisquare test to examine whether a factor improved the model at $\mathrm{p}<.05$. Significant interactions were analyzed using pairwise comparisons only for the comparisons of interest (i.e. simple comparisons in which one factor differed). The p-value was adjusted only for the number of selected tests, using the Benjamini-Hochberg correction. This correction method controls the False Discovery Rate, the expected proportion of false positives, while keeping a high power (Hemmelmann, Horn, Süsse, Vollandt, \& Weiss, 2005).

How each model was built, the model summaries, and emmeans output of relevant significant interactions can be found in Supplementary Materials A for verbs and Supplementary Materials B for objects. The estimates reported in the Results belong to the best-fit model. All best-fit models contained the random effects of Item and Participant, with a random slope for Accent over Participants, and the fixed effects of Context, Accent, ROI, and Group. In addition, the model for verbs (100-200ms) included the interactions Context * ROI, Accent * ROI, Context * Group, Accent * Group, Context * Accent * Group, and Context *Accent * ROI* Group. The model for the verb $(200-390 \mathrm{~ms})$ also included Context $*$ ROI, Context*Group, Context * Accent* Group, ROI* Group, Context * ROI* Group, and Accent $*$ ROI * Group. The model for the objects $(100-200 \mathrm{~ms})$ included the interaction Accent $*$ ROI, Context * Group, Accent * Group, Context * ROI * Group, and Context * Accent * ROI * Group. The model for the objects $(200-390 \mathrm{~ms})$ included Context $*$ ROI, Context * Group, ROI * Group, and Context * Accent * ROI * Group. Finally, the model for objects $(500-900 \mathrm{~ms})$ included Context * ROI, Context * Group, $R O I *$ Group, and Context * Accent * ROI * Group. Because we are interested in how listeners process accentuation (i.e. the effect of Accent) and how the presence of context affects accent processing (i.e. the interaction between Context and Accent), we focused on reporting main effects of and interactions with Accent in the Results. We only examined the highest level of an interaction that included Accent. For example, if a model included significant effects of Accent * ROI * Group and Context $*$ Accent $*$ ROI * Group, we only examined the latter as the additional factor in the interaction may explain the effect of Accent more accurately. By only examining the interactions with Accent, we could eliminate unnecessary analyses and thus lower our experimentwise error rate (Luck \& Gaspelin, 2017).

\section{Results}

\subsection{Semantic relatedness task}

Mean accuracy of the semantic relatedness task was $87.43 \%$ (SD $=4.20 \%)$ for the L1 English group and $90.35 \%$ (SD $=3.83 \%$ ) for the L2 English group. Both groups scored above $80 \%$ accuracy, suggesting that they paid attention to the stimuli.

\subsection{ERP results on the verb}

The ERPs to the verbs from the L1 English group are depicted in Figure 1, and from the L2 English group in Figure 2. Both figures showed that accentuation elicited early responses that started from $100 \mathrm{~ms}$ after word onset and that the responses to the accented verbs were more positive than to the unaccented verbs. There may also be a modifying effect of the context. Figure 3 depicts topographies for an overview of the differences in responses to accentuation between the L1 and L2 English groups. The ERP results tested statistically with our pre-determined time-windows: 100 to $200 \mathrm{~ms}$ for the expectancy of the pitch accent, and 200 to 390ms for the effects of the accentuation. 
L1 English
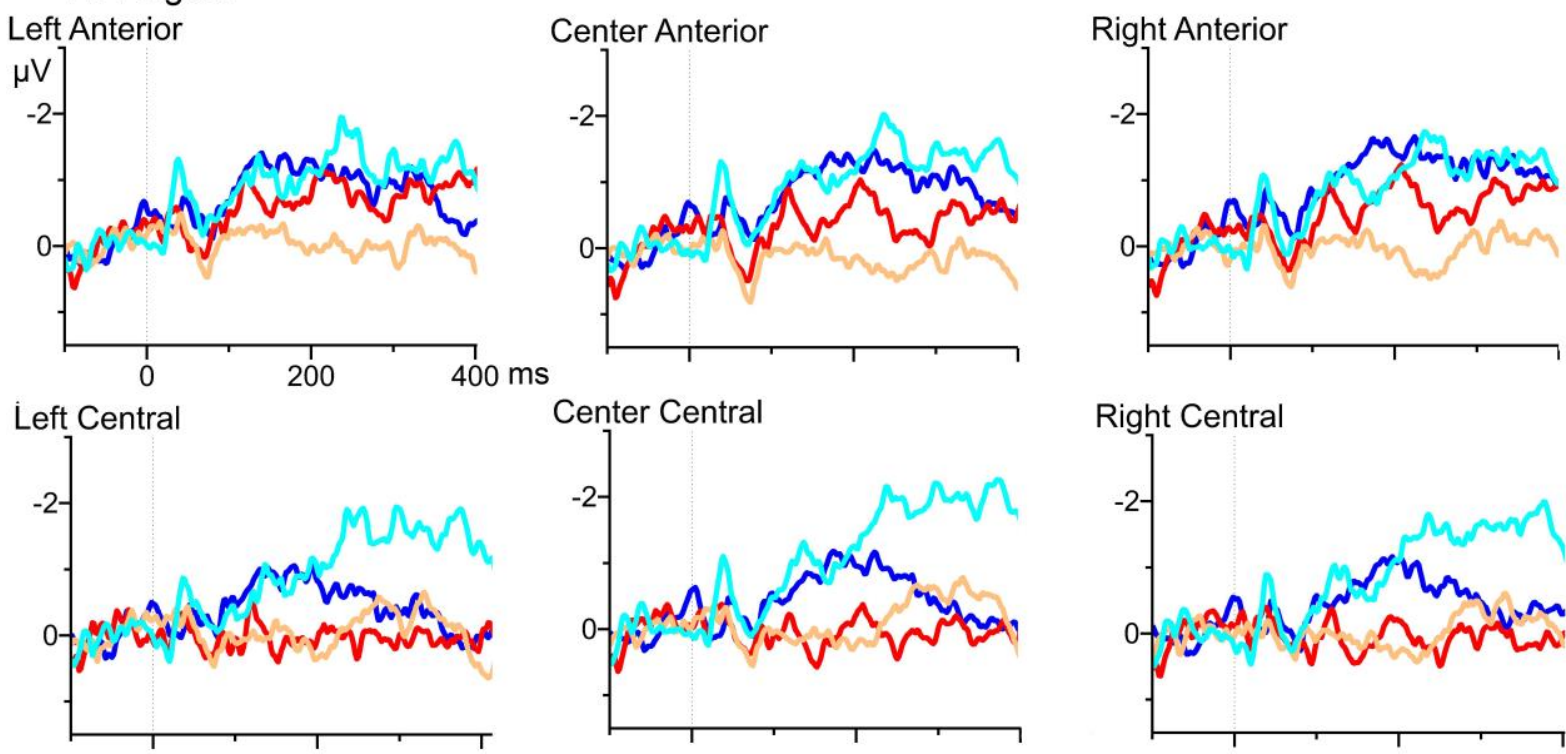

Center Central
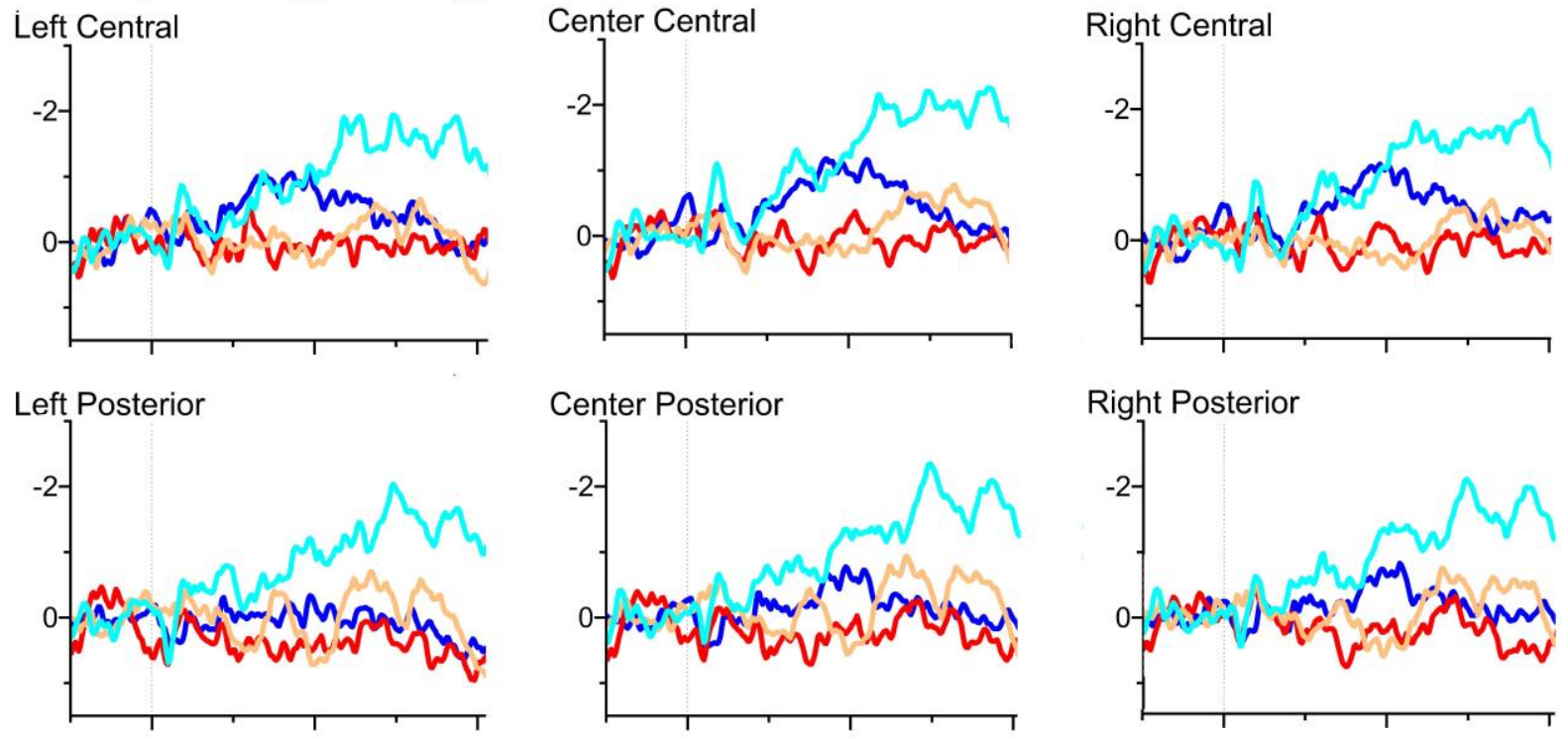

- Accented verbs with context $\quad$ - Unaccented verbs with context

Figure 1. ERPs to the verbs for native speakers of English (L1 English) in nine scalp regions. Red lines depict responses to accented verbs with context; orange lines to accented verbs without context; dark blue lines to unaccented verbs with context; and light blue lines to unaccented verbs without context. 


\section{L2 English}

Left Anterior
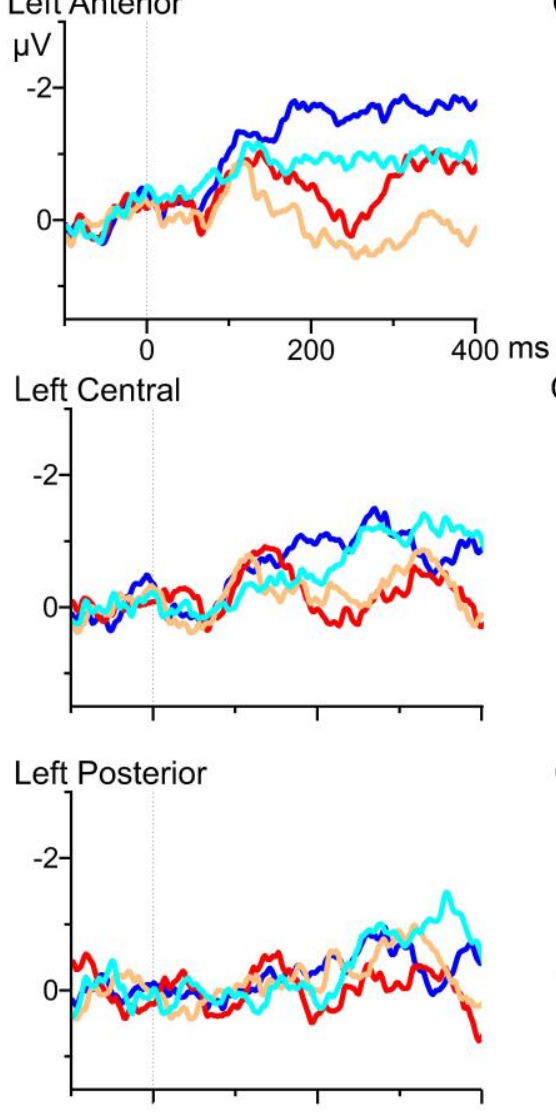

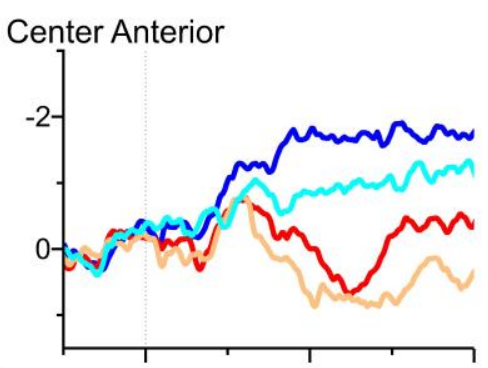

Center Central

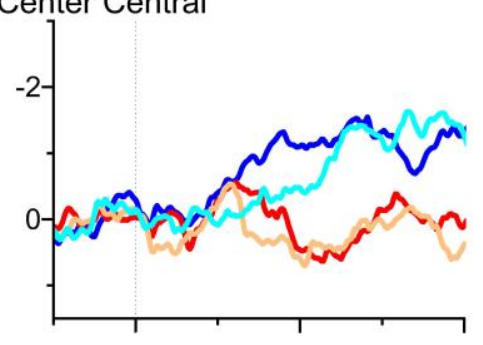

Center Posterior

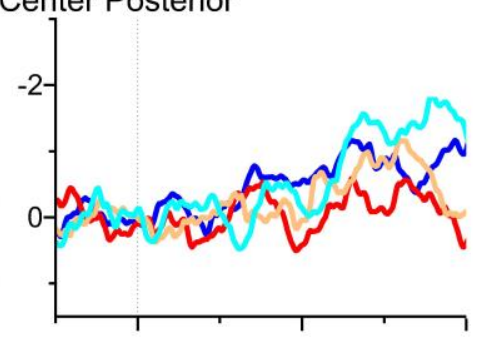

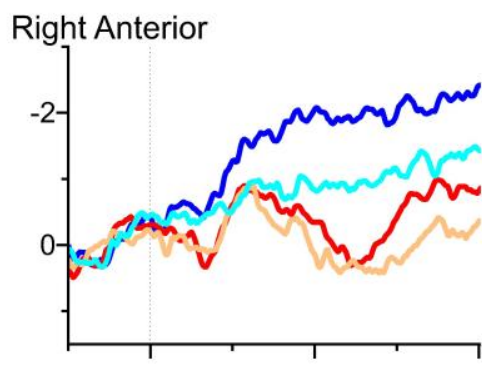
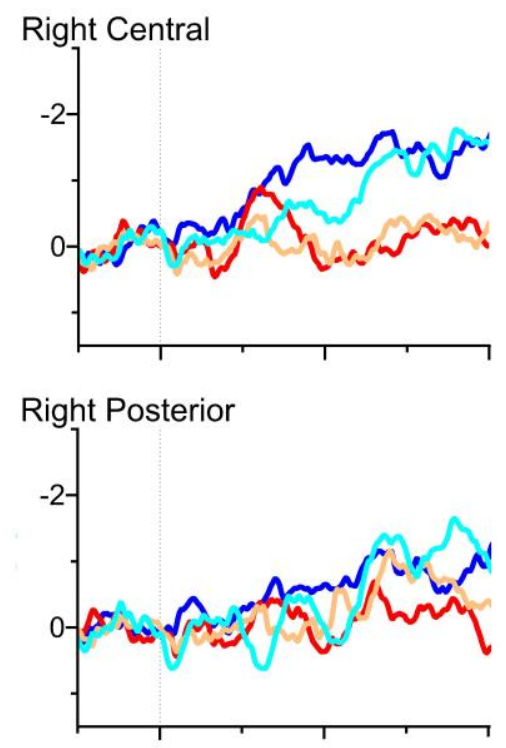

\section{- Accented verbs with context}

- Unaccented verbs with context Accented verbs without context - Unaccented verbs without context

Figure 2. ERPs to the verbs for Dutch learners of English (L2 English) in nine scalp regions. Red lines depict responses to accented verbs with context; orange lines to accented verbs without context; dark blue lines to unaccented verbs with context; and light blue lines to unaccented verbs without context.

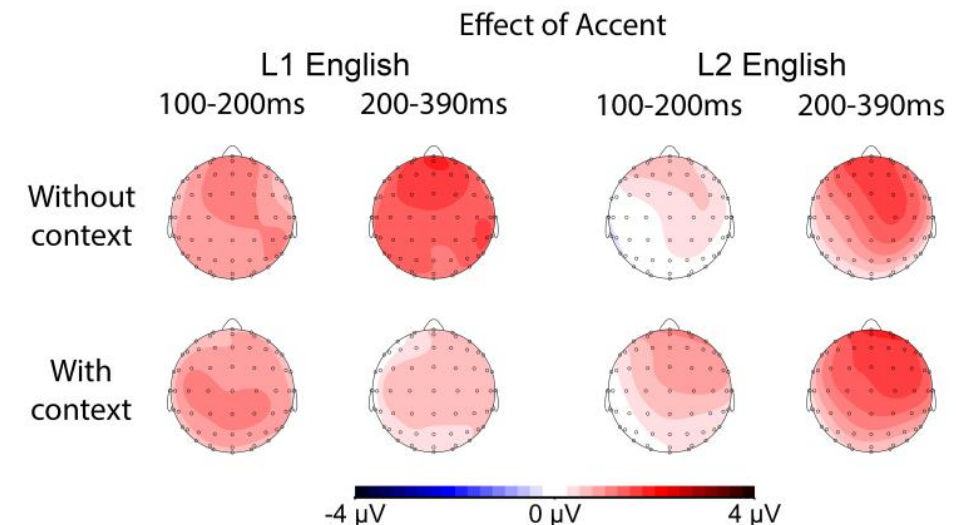

Figure 3. Topography maps for the effect of accent in the two context conditions in the two timewindows 100-200ms and 200-390ms. The maps from the native speakers of English (L1 English) are on the left side and the maps from the Dutch learners of English (L2 English) are on the right side. The topographies were created by subtracting the value from the unaccented verbs in each context condition from the accented verbs in the respective context condition.

\subsubsection{Verb: 100-200ms}

In the best fitting model, we found significant main effects of Intercept, Accent, ROI, and Group, and significant interactions Accent * ROI, Context * ROI, Accent * Group, and Context * Accent * ROI* Group, for details see Supplementary A, of which Context * Accent * ROI * Group was tested further. The L1 English group responded to the accented verbs with more positivity than unaccented verbs in the left and center anterior regions and in the right central region, although this was only significant in the absence of context $(b=1.781, t=3.796, p=.002$ and $b=1.436, t=3.059, p=.017$ for left and 
center anterior respectively, and $b=1.531, t=3.276, p=.010$ for the right central region). This positivity for accented verbs was also found in the L2 English group. However, this was only significant in the center and right anterior region in the presence of context $(b=1.468, t=3.947, p=.002$ and $b$ $=1.523, t=4.097, p=.002$ respectively). Accented verbs therefore elicited an early positive response in both groups, which is in line with our observations and the predictions that expectancy for the pitch accent would result in early positivity. The groups differed on the effect of the context on the positivity. Whereas the L1 English group only showed early positivity to the accentuation in the absence of context, the L2 English group did so in the presence of context. This suggests that the L2 English group used the context differently in their processing of the verb than the L1 English group did. This also goes in line with our predictions that the L2 English group would use contextual information to process prosodic information more easily.

\subsubsection{Verb: $200-390 m s$}

Between 200 to $390 \mathrm{~ms}$, we found significant main effects of Intercept, Context, Accent, ROI, and Group, and significant interactions Context * Group, ROI * Group, Context * ROI * Group, and Accent $*$ ROI * Group. Accent $*$ ROI * Group was examined further with pairwise comparisons, for details see Supplementary A. Again, the statistical analyses are in line with our observations; both groups responded with more positivity to accented verbs than unaccented verbs. In the L1 English group, this was significant in the three anterior regions, and in the center and right central regions $(b=1.756, t=$ $4.463, p=.001 ; b=1.178, t=2.996, p=.006$; and $b=1.136, t=2.889, p=.002$ for left, center, and right anterior regions respectively, and $b=1.153, t=2.930, p=.006$ and $b=1.264, t=3.212, p=.004$ for center and right central regions respectively). In the L2 English group, this was the case for all regions except for the left posterior region $(b=0.783, t=2.536, p=0.015 ; b=1.007, t=3.266, p=$ 0.004 ; and $b=1.124, t=3.642, p=0.002$ for left, center, and right anterior regions respectively, $b=$ $1.403, t=4.554, p=0.001 ; b=1.034, t=3.359, p=0.004$; and $b=1.094, t=3.553, p=0.002$ for left, center, and right central regions respectively, and $b=0.921, t=2.997, p=0.006$ and $b=0.835, t$ $=2.714, p=0.010$ for center and right posterior regions). Therefore, both groups showed a broadly distributed positive response to the accentuation of the verb, which is in line with our predictions of the 'accent positivity'.

\subsubsection{Summary of the effects at the verb}

The results from the verb showed that there are similarities and differences in the prosodic processing of L1 and L2 English listeners. The L1 English group showed an early positive expectancy pattern for the pitch accent adjacent to only in isolated sentences, extending over the left-center anterior and right central scalp regions. This was followed by an anterior-central distributed 'accent positivity', which was independent of the presence of the context. The L2 English group also showed an expectancy pattern for adjacent accentuation, which was distributed over the center-right anterior scalp regions but was only present if preceded by a context. Similar to the L1 English group, the L2 English group responded with a broadly distributed 'accent positivity' to the pitch accent. For both the L1 and L2 English groups, this early positive expectancy and the 'accent positivity' is similar to what was found in L1 Dutch prosodic processing patterns (Dimitrova, 2012).

\subsection{ERP results on the object}

The ERPs to the objects are presented in Figure 4 for the L1 English group and Figure 5 for the L2 English group. Both figures showed that accentuation elicited a negativity in the anterior regions in both groups starting from approximately $300 \mathrm{~ms}$ after word onset. However, the figures showed more differences than similarities in how objects were processed. Figure 6 depicts topographies for an overview of the differences in responses to accentuation between the L1 and L2 English groups. Responses to the objects were tested statistically in the pre-determined windows: 100 to $200 \mathrm{~ms}$ for the expectancy of the pitch accent, 200 to $390 \mathrm{~ms}$ for the effects of the pitch accent, and 500 to $900 \mathrm{~ms}$ for the re-interpretation of the sentence. 


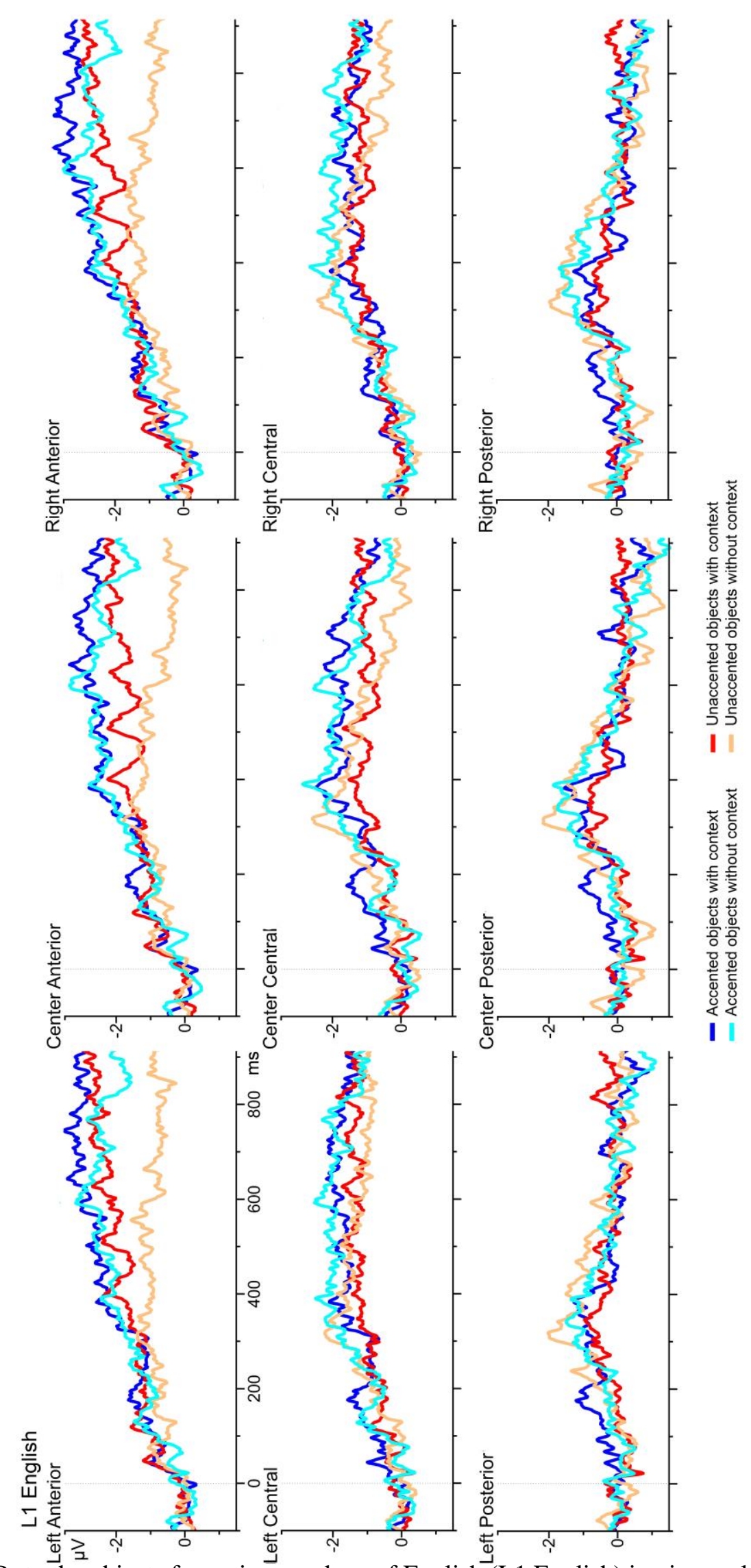

Figure 4. ERPs to the objects for native speakers of English (L1 English) in nine scalp regions. Dark blue lines depict responses to accented objects with context; light blue lines to accented objects without context; red lines to unaccented objects with context; and orange lines to unaccented objects without context. 


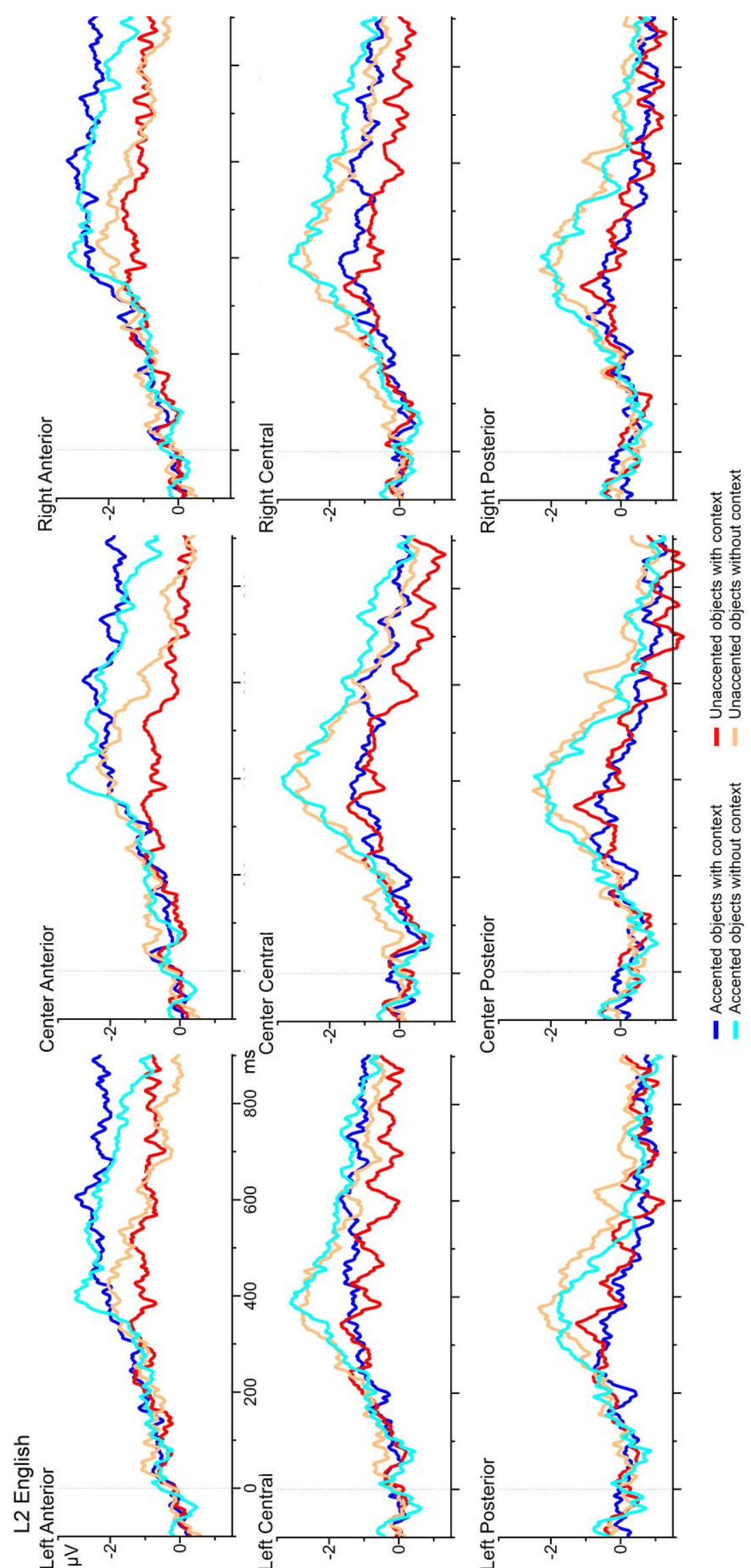

Figure 5. ERPs to the objects for Dutch learners of English (L2 English) in nine scalp regions. Dark blue lines depict responses to accented objects with context; light blue lines to accented objects without context; red lines to unaccented objects with context; and orange lines to unaccented objects without context. 


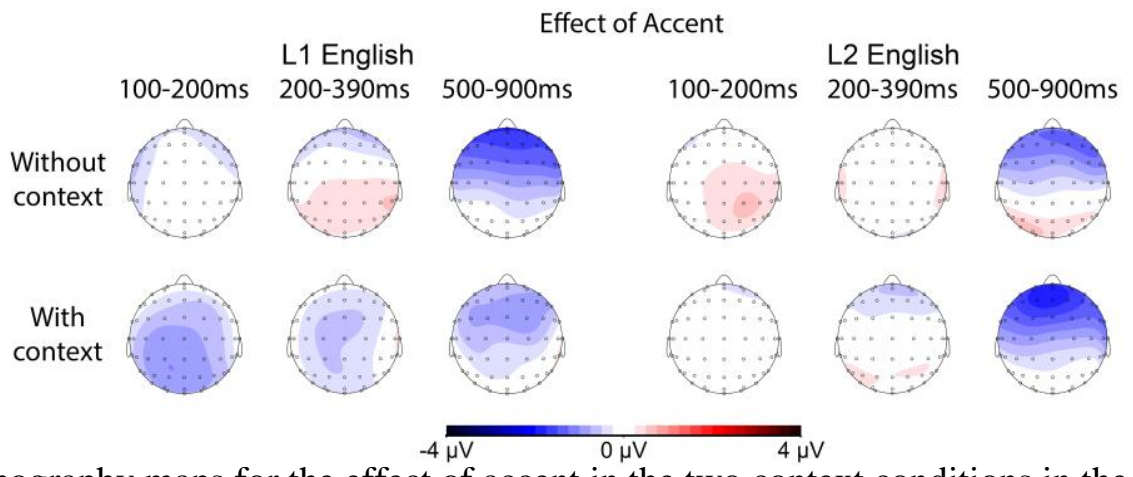

Figure 6. Topography maps for the effect of accent in the two context conditions in the three timewindows $100-200 \mathrm{~ms}, 200-390 \mathrm{~ms}$, and $500-900 \mathrm{~ms}$. The maps from the native speakers of English (L1 English) are on the left side and the maps from the Dutch learners of English (L2 English) are on the right side. The topographies were created by subtracting the value from the unaccented objects in each context condition from the accented objects in the respective context condition.

\subsubsection{Object: $100-200 \mathrm{~ms}$}

We found significant main effects of Accent and ROI and significant interactions Accent * ROI, Accent * Group, Context * ROI* Group, and Context * Accent* ROI* Group, also noted in Supplementary $\mathrm{B}$, for which the interaction Context * Accent * ROI * Group was examined further. However, after adjustment for pairwise comparisons in the post hoc tests, there were no significant effects of accentuation in neither group in any of the scalp regions. This suggests that neither the L1 nor L2 English group showed early responses to the pitch accent and did not have expectations for nonadjacent accentuation. This finding goes against our predictions that nonadjacent accentuation would elicit an early negativity.

As the four-way interaction was not significant, we decided to inspect the two-way interactions Accent * Group and Accent * ROI, and the main effect of Accent to see if we could find any evidence for the effect of accentuation. However, in both two-way interactions, the effect of Accent remained insignificant, even before p-value adjustments. The main effect of Accent in the model does however suggest that accented objects elicited more negativity than unaccented objects $(b=-2.091, t=3.088, p$ $=0.002$ ). There may be an effect of accentuation, eliciting more early negativity and suggesting that listeners responded to the pitch accent early. However, we remain careful with this conclusion as there were insignificant interactions that may have provided different results.

\subsubsection{Object: $200-390 \mathrm{~ms}$}

As can also be observed between 200 to 390ms in Figure 4 and 5, we did not find significant main effects and interactions that included the effect of Accent in the final model. Instead, we found significant effects $R O I$ and $R O I *$ Group. This time-window was therefore not examined any further. The lack of significant Accent effects goes against our predictions that both groups would show the 'accent positivity' as a response to the pitch accent, suggesting that the groups did not perceive the pitch accent.

\subsubsection{Object: $500-900 \mathrm{~ms}$}

We found significant main effects Accent and ROI and the interactions Context * Group, ROI * Group, and Context * Accent * ROI* Group; details can be found in Supplementary B. We analyzed the significant interaction Context * Accent * ROI * Group further. Despite the significant four-way interaction in the final model, after adjustments for multiple comparisons in the post-hoc tests, there were no significant effects of Accent in either group in any of the nine scalp regions. This finding is not in line with our prediction that accentuation in the object would result in sentence re-interpretation in the L1 English group. However, we predicted that the L2 English group would not show such an effect.

The main effect of Accent in the final model suggested that the accented objects elicited more negative responses than unaccented objects $(b=-1.208, t=2.262, p=0.024)$, although this effect may be skewed due to the presence of interactions.

\subsubsection{Summary of the effects at the object}

The lack of results from the object suggested that accentuation nonadjacent to only did not elicit prosodic processing in the L1 and L2 English groups. There was not an expectation for the pitch accent nor did listeners respond to the acoustic features of the pitch accent, or did they re-interpret the sentence. These results deviate from our predictions.

\section{Discussion}


The current study examined how native speakers of English and advanced Dutch learners of English differ in their integration of prosodic cues with semantic and syntactic information in sentences with only. By doing so, our goal was to provide evidence for the underlying mechanism for the difficulties that these L2 English listeners have shown to experience during real-time processing of sentences with only (Ge et al., in prep). We hypothesized that L2 English listeners may experience influence from the L1 or may fail to integrate the prosodic-semantic-syntactic interface of such sentences. To map rapid integration processes, we used an ERP paradigm to measure neural responses as pitch accents unfolded. These pitch accents were either on verbs or on objects, embedded in sentences with only that were either presented in isolation or with contextual sentences. These context sentences provided alternatives to actions and objects, and provided information of an upcoming contrast to ease prosodic processing. By using an ambiguous context, the accentuation in the target sentence with only was the one cue to the location of the contrastive focus. Sentence constructions were always the same, resulting in verbs being adjacent to only and objects nonadjacent to only. Accentuation of the object therefore resulted in prosodic focus nonadjacent to only, which is a construction not commonly used by Dutch speakers in their L1. Based on previous L1 Dutch processing of contrastive prosody in sentences with and without only (Dimitrova, 2012; Dimitrova et al., 2012), we expected that English listeners would show an early positive expectancy peak, followed by a broadly distributed 'accent positivity' for adjacent accentuation, and a negative frontal expectancy peak, 'accent positivity', and the P600 for nonadjacent accentuation. Dutch listeners would fail to show such expectancy and P600 patterns. In addition, they would use the context to facilitate their prosodic processing. Our results were partly in line with our predictions, but they also showed unexpected findings. These will be discussed below.

\subsection{Processing of the verb adjacent to only}

Both the L1 and L2 English groups displayed similar responses in the expectancy time-window: an early positivity to the pitch accent between 100 to $200 \mathrm{~ms}$. We interpreted this finding as both groups being able to include prosodic information in their early evaluation of the verb. Furthermore, given that the positivity occurred shortly after word onset, we considered the responses to be facilitated by prior expectations for the pitch accent. This in line with our predictions for the L1 English group and what has been found for L1 Dutch processing of pitch accent adjacent to only (Dimitrova, 2012). However, we predicted that the L2 English group would not show such an expectancy effect as they have shown to be delayed in their use of prosodic information in the interpretation of the sentence meaning (Ge et al., in prep), suggesting an inability to predict prosodic focus. Instead, the results from the current study suggest that the L2 English listeners are capable of doing so when the focal word is the verb.

Our findings on the modifying effect of the context may elaborate on this unexpected L2 finding. We found differences in how context affected prosodic processing in each group. We found that the early positivity was only present in the L2 English group when the verbs were preceded by the context. This suggests that the L2 listeners may have only been able to respond to the pitch accent so quickly when they had additional information from the context. By presenting alternatives and signaling an upcoming contrast in the context, the listeners did not have to mentally generate this information at the moment they heard the contrast; it was already primed. It is plausible that the listener was then simply waiting for a cue to the exact placement of the contrast, e.g. only. When the L2 listeners heard only, their expectation for contrast may have automatically been projected to the word most likely to be contrasted. Based on their L1 experience, this would be the word adjacent to only. If our reasoning is correct, we could apply a similar rationale for the findings in the L1 English group. L1 English listeners only showed the early positivity when accented verbs were presented in isolated sentences and not in context. The context provided alternatives for both the verb and the object, which may have been a cue to them that the contrast may not necessarily be adjacent to only, resulting in a diminished, nonsignificant effect in the sentences with context. Without context, the listeners may have defaulted the contrast to the verb.

In addition to this early effect, we found that the accentuation elicited a positivity between 200 to $390 \mathrm{~ms}$ that was widely distributed over the scalp in both groups. This is in agreement with our predictions of the 'accent positivity': both groups were sensitive to the accentuation adjacent to only. However, this 'accent positivity' occurred independent of the context condition, suggesting that the groups could process the pitch accent without the contextual information.

All in all, adjacent accentuation elicited processes that was generally similar between L1 and L2 English listeners. Both groups expected the pitch accent and responded to its acoustic features. These processing patterns have also been found for L1 Dutch processing of contrastive pitch accents in sentences with only (Dimitrova, 2012). This suggest that these contrastive pitch accents are processed similarly by L1 English, L2 English, and L1 Dutch listeners.

\subsection{Processing of the object nonadjacent to only}

Nonadjacent accentuation was seemingly more difficult to process than the adjacent accentuation as we did not find the expected prosodic processing patterns. The lack of findings between 100 and $200 \mathrm{~ms}$ suggest that neither the L1 nor the L2 English listeners were expecting the accentuation. However, the main effect of accentuation did suggest that accented objects elicited more negativity than unaccented objects and that therefore there was early processing of the pitch accent. However, due to the absence of findings in the ROIs, in the groups, or in the context conditions, we cannot conclude whether it is an expectancy effect, located in the frontal scalp regions, if there are group differences, or if there is an effect of context.

The lack of findings of accentuation effects between 200 to 390ms suggest that both the L1 and L2 English listeners did not respond to the acoustic features of the pitch accent on the object. A possible explanation could be that the listeners were simply not sensitive to the pitch accent and did not hear it. 
However, the eye-tracking data by Ge et al. (in prep.) showed that both L1 and L2 English listeners referred to the picture that showed the alternative to the object-focus, implying that they did hear the pitch accent and were able to adjust their interpretation of the sentence accordingly. We can therefore conclude that it was not the lack of sensitivity to the accentuation. An alternative explanation could be that the listeners already adjusted their expectations at the moment they heard the verb. Based on the accentuation of the verb, the listeners could already predict how the rest of the sentence would proceed; If the verb was accented, it meant that the object was not and if the verb was unaccented, the object was. After hearing the verb, the listeners may have not needed the additional prosodic information from the object, resulting in the lack of processing of the accent on the object. This is supported by observations in the waveforms in Figure 4 and 5, it seemed that objects were overall processed differently than the verbs.Figure 4 did not show any accentuation effects in the L1 English group and Figure 5 shows a clear negative peak around $400 \mathrm{~ms}$ in the L2 English group for objects in isolated sentences compared to sentences with context. We did not statistically analyze this effect as we had pre-determined our timewindows but this peak suggests that the L2 English listeners responded to the object based on whether it was preceded by the context, suggesting lexical processing rather than prosodic processing. We will propose a reason of why this peak was present. The orientation and peak latency suggests that this peak represents the N400, which has previously been associated with difficulty to integrate semantic information into the sentence meaning (Brown \& Hagoort, 1993). The N400 only occurred in the absence of the context, suggesting that the L2 listeners had difficulties accepting the objects in isolated sentences but not when it was preceded by the context. Based on the modifying effect of context in the processing of the verb that we found in L2 English listeners, we propose that the L2 English listeners use the context to anticipate the object, whereas in isolated sentences these objects come as a surprise. We took another look at our stimuli and we found 8 sets of sentences $(n=32)$ that have strange combinations of actions with objects (e.g. The dinosaur is only brushing the sweater) that the L2 English listeners may not have accepted. Although this is a relatively small number compared to the overall 240 experimental stimuli that the listeners were presented with, it is a possibility that these 24 stimuli elicited a stronger effect than the pitch accent, which resulted in the N400 for infelicitous combinations. This, however, does not explain why we did not find such an effect in the L1 English group.

Finally, in the time-window 500-900ms we did not find evidence for the P600 in the post-hoc tests. However, the final model for this time-window and Figure 3 and 4 did suggest an effect of accentuation in the anterior regions, in which accented objects may have resulted in more negativity than unaccented objects in both groups. This suggests that the pitch accent elicited a smaller P600, resulting in easier sentence re-analysis in both groups (Burkhardt, 2007). Again, we should accept this theory with caution as the interaction was not significant in the post hoc tests.

In general, nonadjacent accentuation elicited similar processes in both groups. The listeners did not expect nor respond to the acoustic features of the pitch accent and may or may not have re-interpreted the sentence similarly based on the pitch accent. These findings are different from what was found for L1 Dutch processing of contrastive pitch accents in sentences with only (Dimitrova, 2012), which suggests that L1 and L2 English listeners process contrastive pitch accents that are placed nonadjacent to only differently from L1 Dutch.

\subsection{Underlying mechanisms}

Considering we only found anticipation and processing of the pitch accent at the verb but not at the object, we propose that the listeners experienced difficulties processing nonadjacent accentuation. The facilitatory effect of the context in their verb processing and the suspected N400 effect found in the object, it may be that their overall processing strategy was different to that of the L1 English listeners, being guided more by lexical processing (also found in Lee et al., 2019). As the L2 English processing pattern is similar to the L1 English and L1 Dutch pattern on the verb, but dissimilar on the object, we conclude that L2 English listeners may experience difficulty adjusting their expectations for prosodic focus. It is as if they considered the verb to be the contrast, but were unable to adjust their interpretation of the contrast to the object when the verb was unaccented. These findings provide additional evidence for the failure to integrate point of view.

\section{Conclusion}

In conclusion, the present study focused on the differences and similarities of L1 and L2 processing of the prosodic-semantic-syntactic interface in English sentences with only by L1 English listeners and advanced Dutch learners of English. Our results suggests that L2 English listeners had a default interpretation of sentences with only, but were unable to adjust their processing strategies when this default interpretation did not hold up. They failed to integrate the information provided at the verb to update and expect upcoming prosodic information at the object.
Abbreviations
The following abbreviations were used to distinguish the two groups:
L1 English: native speakers of English
L2 English: Dutch learners of English

\section{Additional files}

The following Supplementary materials are provided for this article.

Supplementary A 
A PDF file containing the models, final model summary, and emmeans output for the analyses of the verb.

Supplementary B

A PDF file containing the models, final model summary, and emmeans output for the analyses of the object.

\section{Acknowledgements}

We thank all the participants for their participation, and Zenghui Liu, Aelish Hart, and Nelleke Jansen for their help in conducting the experiment. We also thank Piet van Tuijl for his input on the statistical analyses and the technicians of the UiL-OTS for all their technical support, in particular Chris van Run, who helped in troubleshooting and finalizing the necessary scripts to run the experiment. This research was funded by the Utrecht University's Utrecht Institute of Linguistics OTS, the Utrecht University's Graduate School of Life Sciences, and by the Westerdijk Talent grant awarded to Aoju Chen by the Netherlands Organisation for Scientific Research (NWO).

\section{Competing interests}

The authors declare that there were no competing interests during this research.

\section{Authors' contributions}

Rachida Ganga, Marijn E. Struiksma, and Aoju Chen designed the study. Rachida conducted the experiment, pre-processed and analyzed the data, and wrote the article. Marijn proposed steps during the data pre-processing and analysis and provided detailed feedback on the statistical analyses and the article. Aoju Chen supervised the experiment and provided feedback during each step of the production of this article. Haoyan Ge and Virginia Yip provided stimuli and their consent to base the current experiment on their previous experiments, and provided feedback on the article.

\section{References}

Akker, E., \& Cutler, A. (2003). Prosodic Cues to Semantic Structure in Native and Nonnative Listening. Bilingualism: Language and Cognition, 6, 81-96. https://doi.org/10.1017/S1366728903001056

Bates, D., Maechler, M., Bolker, B., \& Walker, S. (2015). Fitting Linear Mixed-Effects Models Using lme4. Journal of Statistical Software, 67, 1-48. https://doi.org/10.18637/jss.v067.i01

Boersma, P., \& van Heuven, V. (2001). Speak and unSpeak with Praat. Glot International, 5(9-10), 341-347.

Bouma, G., Hendriks, P., \& Hoeksema, J. (2007). Focus Particles Inside Prepositional Phrases: A Comparison of Dutch, English, and German. Journal of Comparative German Linguistics, 10, 1-24. https://doi.org/10.1007/s10828-006-9006-1

Brown, C., \& Hagoort, P. (1993). The Processing Nature of the N400 : Evidence from Masked Priming. Journal of Cognitive Neuroscience, 5, 34-44. https://doi.org/10.1162/jocn.1993.5.1.34

Burkhardt, P. (2007). The P600 Reflects Cost of New Information in Discourse Memory. Cognitive Neuroscience and Neuropsychology, 18, 1851-1854. https://doi.org/10.1097/WNR.0b013e3282f1a999

Chen, A., \& Lai, V. (2012). Comb or Coat: The Role of Intonation in Online Reference Resolution in a Second Language. Sound and Sounds Utrecht, 57-68.

Clahsen, H., \& Felser, C. (2006). How native-like is non-native language processing? Trends in Cognitive Sciences, 10(12), 564-570. https://doi.org/10.1016/j.tics.2006.10.002

Dimitrova, D., Stowe, L., \& Hoeks, J. (2015). When Correction Turns Positive: Processing Corrective Prosody in Dutch. PLoS One, 10, e0126299. https://doi.org/10.1371/journal.pone.0126299

Dimitrova, D. V. (2012). Neural correlates of prosody and information structure (University of Groningen). Retrieved from http://www.rug.nl/research/portal/files/2434008/DVDimitrova_thesis_20120507.pdf

Dimitrova, D. V, Stowe, L. A., Redeker, G., \& Hoeks, J. C. J. (2010). Focus Particles and Prosody Processing in Dutch: Evidence from ERPs. Speech Prosody, 1-4. Chicago.

Dimitrova, D. V, Stowe, L. A., Redeker, G., \& Hoeks, J. C. J. (2012). Less Is Not More: Neural Responses to Missing and Superfluous Accents in Context. Journal of Cognitive Neuroscience, 24, 2400-2418. 
Filik, R., Paterson, K. B., \& Liversedge, S. P. (2009). The influence of only and even on online semantic interpretation. Psychonomic Bulletin and Review, 16, 678-683. https://doi.org/10.3758/PBR.16.4.678

Foolen, A., Gerrevink, R. Van, Hogeweg, L., \& Prawiro-Atmodjo, P. (2009). The placement of focus particles in Dutch. Linguistics in the Netherlands, 26(April 2016), 51-63. https://doi.org/10.1075/avt.26.06foo

Frenck-Mestre, C. (2002). An on-line look at sentence processing in the second language. In J. Altarriba \& R. Herridia (Eds.), Bilingual Sentence Processing. North Holland.

Ge, H., Chen, A., \& Yip, V. (2018). L1 Effects on L2 comprehension of focus-to-prosody mapping: A comparison between Cantonese and Dutch learners of English. 9th International Conference on Speech Prosody 2018, 187-191. https://doi.org/10.21437/speechprosody.2018-38

Ge, H., Mulders, I., Yip, V., \& Chen, A. (in prep.). Processing multiple interfaces in native and nonnative speakers of English: A visual world eye-tracking study of focus in sentences with "only."

Gussenhoven, C. (1983). On the Grammar and Semantics of Sentence Accents. Dordrecht: Floris.

Hemmelmann, C., Horn, M., Süsse, T., Vollandt, R., \& Weiss, S. (2005). New concepts of multiple tests and their use for evaluating high-dimensional EEG data. Journal of Neuroscience Methods, 142, 209-217. https://doi.org/10.1016/j.jneumeth.2004.08.008

Juffs, A. (2005). The influence of first language on the processing of wh-movement in English as a second language. Second Language Research, 21(2), 121-151. https://doi.org/10.1191/0267658305sr255oa

Ladd, D. R. (1980). The structure of intonational meaning: Evidence from English. Bloomington: Indiana University Press.

Lee, A., Perdomo, M., \& Kaan, E. (2019). Native and second-language processing of contrastive pitch accent: An ERP study. Second Language Research. https://doi.org/10.1177/0267658319838300

Lemhöfer, K., \& Broersma, M. (2012). Introducing LexTALE: A quick and valid Lexical Test for Advanced Learners of English. Behavior Research Methods, 44(2), 325-343. https://doi.org/10.3758/s13428-011-0146-0

Luck, S. J., \& Gaspelin, N. (2017). How to get statistically significant effects in any ERP experiment (and why you shouldn't). Psychophysiology, 54, 146-157. https://doi.org/10.1111/psyp.12639

Marinis, T., Roberts, L., Felser, C., \& Clahsen, H. (2005). Gaps in second language sentencep rocessing. Studies in Second Language Acquisition, 27, 53-78. https://doi.org/10.1017/S0272263105050035

Mulders, I., \& Szendroi, K. (2016). Early association of prosodic focus with alleen "only": Evidence from eye movements in the visual-world paradigm. Frontiers in Psychology, 7, 150. https://doi.org/10.3389/fpsyg.2016.00150

Regel, S., Meyer, L., \& Gunter, T. C. (2014). Distinguishing neurocognitive processes reflected by P600 effects: Evidence from ERPs and neural oscillations. PLoS ONE, 9, e96840. https://doi.org/10.1371/journal.pone.0096840

Rooth, M. (1992). A theory of focus interpretation. Natural Language Semantics, 1, 75-116.

Russell Lenth. (2019). emmeans: Estimated Marginal Means, aka Least-Squares Means. R package version 1.3.5. Retrieved from https://cran.r-project.org/package=emmeans

Sorace, A. (2011). Pinning down the concept of "interface" in bilingualism. Linguistic Approaches to Bilingualism, 1, 1-33. https://doi.org/10.1075/lab.1.1.01 sor

Sorace, A., \& Filiaci, F. (2006). Anaphora resolution in near-native speakers of Italian. Second Language Research, 22(3), 339-368. https://doi.org/10.1191/0267658306sr271oa

White, L. (2011). Second language acquisition at the interfaces. Lingua, 121, 577-590. https://doi.org/10.1016/j.lingua.2010.05.005

Zimmermann, M., \& Onea, E. (2011). Focus Marking and Focus Interpretation. Lingua, 121, 16511670. https://doi.org/10.1016/j.lingua.2011.06.002 